\title{
Single and pair neutron transfers at sub-barrier energies
}

\author{
L. Corradi, ${ }^{1}$ S. Szilner,,${ }^{1,4}$ G. Pollarolo, ${ }^{3}$ G. Colò, ${ }^{7}$ P. Mason, ${ }^{2}$ E. Farnea, ${ }^{2}$ E. Fioretto, ${ }^{1}$ A. Gadea,${ }^{8}$ F. Haas, ${ }^{6}$ \\ D. Jelavić-Malenica, ${ }^{4}$ N. Mărginean,${ }^{5}$ C. Michelagnoli, ${ }^{1}$ G. Montagnoli, ${ }^{2}$ D. Montanari, ${ }^{2}$ F. Scarlassara, ${ }^{2}$ N. Soić, ${ }^{4}$ \\ A. M. Stefanini, ${ }^{1}$ C. A. Ur, ${ }^{2}$ and J. J. Valiente-Dobón ${ }^{1}$ \\ ${ }^{1}$ Istituto Nazionale di Fisica Nucleare, Laboratori Nazionali di Legnaro, I-35020 Legnaro, Italy \\ ${ }^{2}$ Dipartimento di Fisica, Università di Padova, and Istituto Nazionale di Fisica Nucleare, I-35131 Padova, Italy \\ ${ }^{3}$ Dipartimento di Fisica Teorica, Università di Torino, and Istituto Nazionale di Fisica Nucleare, I-10125 Torino, Italy \\ ${ }^{4}$ Ruder Bošković Institute, HR-10001 Zagreb, Croatia \\ ${ }^{5}$ Horia Hulubei National Institute of Physics and Nuclear Engineering, R-077125, Bucharest-Magurele, Romania \\ ${ }^{6}$ Institut Pluridisciplinaire Hubert Curien, CNRS-IN2P3, Université de Strasbourg, F-67037 Strasbourg, France \\ ${ }^{7}$ Dipartimento di Fisica, Universitá degli Studi di Milano and Istituto Nazionale di Fisica Nucleare, I-20133 Milano, Italy \\ ${ }^{8}$ IFIC, CSIC-Universidad de Valencia, E-46071 Valencia, Spain
}

(Received 27 May 2011; published 6 September 2011)

\begin{abstract}
Multinucleon transfer cross sections in the ${ }^{96} \mathrm{Zr}+{ }^{40} \mathrm{Ca}$ system have been measured, in inverse kinematics, at bombarding energies ranging from the Coulomb barrier to $\sim 25 \%$ below. Targetlike recoils have been identified in A, $\mathrm{Z}$ and velocity with the large solid angle magnetic spectrometer PRISMA. The experimental data for oneand two-neutron transfer channels have been compared with semiclassical microscopic calculations. For the two-neutron transfer channels the relevance of the transitions to the ground state and to the $0^{+}$excited states of ${ }^{42} \mathrm{Ca}$ are discussed by employing, for the reaction mechanism, the successive approximation. It is found that the transition to the $0^{+}$state at $\sim 6 \mathrm{MeV}$, whose wave function is dominated by the two neutrons in the $2 p_{3 / 2}$ shell, is much larger than the ground state one. The comparison with the inclusive data reveals that transitions to states with high multipolarity and non-natural parity are important. This suggests that more complex two-particle correlations have to be incorporated in the treatment of the transfer process.
\end{abstract}

DOI: 10.1103/PhysRevC.84.034603

PACS number(s): 25.70.Hi, 29.30.Aj, 24.10.-i

\section{INTRODUCTION}

Nucleon transfer reactions at energies close to the Coulomb barrier always played an important role for nuclear structure and reaction dynamics. Two-particle transfer reactions are used for the study of particle-particle correlation in nuclei. For very light ions the outcome of these reactions has been crucial for the formulation of the concept of pairing vibrations and rotations that allowed to correlate the $0^{+}$excited states in neighboring even-even nuclei [1]. With heavy ions multiple transfer of nucleons becomes available in the reaction, providing the possibility of studying the relative role of single-particle and pair-transfer modes. Of particular interest is still whether it is possible to reach a situation where multiple transfer of pairs is dominating the exchange of mass and charge between the interacting nuclei (Josephson effect [2]).

In spite of extensive experimental and theoretical efforts we are still missing a detailed understanding of multiple transfer processes, for instance, it is not clear whether and how one can probe pair transfer degrees of freedom. This, in part, is because most of the existing studies involve inclusive cross sections that have been obtained at above the Coulomb barrier, where interpretation of the data is made difficult by the presence of several competing processes that depopulate the channels of interest. The problematics connected with the pair correlations is of current interest in ongoing research with radioactive beams, where, for example, the pairing interaction is expected to be significantly modified in nuclei with extended neutron distributions [3]. The recent works on ${ }^{11} \mathrm{Li}$-induced reactions [4] provided evidence of phonon-mediated pairing interaction [5].
In this paper we are dealing with rearrangement collisions $A(a, b) B$ at energies below the Coulomb barrier. Our attention is focused on reactions that involve the transfer of neutrons only. Even though the cross sections are much smaller than the ones encountered at higher energies, certain advantages appear when dealing with energies for which the $a+A$ nuclei cannot overcome their mutual Coulomb barrier. In this situation the two nuclei are kept apart, making negligible the formation of compound nuclei and ensuring that the transfer process is a direct one. At the same time the distortion of the Coulomb elastic waves by the nuclear attraction is very small and may easily be accounted for [6]. The main advantage, however, is that in the calculations of the transfer probabilities one needs only the overlap of the tails of the intrinsic wave functions that are involved in the transfer process, and these asymptotic behaviors are well known at least for singleparticle transfer.

The angular distributions of sub-Coulomb transfer reactions are featureless with a behavior that is independent on the transferred angular momentum $\ell$; these angular distributions grow monotonically, reaching the maximum at $\theta_{\text {c.m. }}=180^{\circ}$. For each scattering angle $\theta$ the radial dependence of the contribution for the transfer peaks at the distance of closest approach corresponding to the Coulomb trajectory leading to that scattering angle. This localization is particularly marked for heavy ions because of the very short wavelength. It is this localization that makes plausible the use of semiclassical approximations [7-9], where the relative motion is considered classical and the transfer process is treated quantum mechanically. It is only at very low bombarding energy and for not too 
heavy systems that the quantal penetrability may be important so as to invalidate the semiclassical approximation.

Following the above considerations, in order to have an overall description of the experimental data on multinucleon transfer reactions it is convenient to extract the transfer probability $P_{t r}$, defined as the ratio of the transfer cross section to the corresponding Rutherford cross section,

$$
P_{t r}=\frac{d \sigma_{t r}}{d \sigma_{\text {Ruth }}},
$$

and to plot this transfer probability as a function of the distance of closest approach $D$ for a Coulomb trajectory, which is related to the center-of-mass scattering angle $\theta_{\text {c.m. }}$ via the expression

$$
D=\frac{Z_{a} Z_{A} e^{2}}{2 E_{\text {c.m. }}}\left(1+\frac{1}{\sin \left(\theta_{\text {c.m. }} / 2\right)}\right)
$$

The extraction of the transfer probability $P_{t r}$ is very attractive since the angular distributions obtained at different bombarding energies will be all superimposed, provided the $Q$-value window does not change very much with the bombarding energy (see below). We notice also that the transfer probability at large distances $D$ may be obtained by measuring an excitation function at fixed backward center of mass angle or by measuring angular distributions at forward angles.

The conditions that are met at energies below the barrier are also very convenient from theoretical point of view. In fact, at these energies one has been able to obtain a nice description of the data also for the two-particle transfer channel by including contributions coming from the successive mechanism [10-12], while the one-nucleon transfer is conveniently described by summing the contributions, calculated in the simple first-order Born approximation, of all the open channels.

In literature many data on transfer reactions have been represented via the transfer probability $P_{t r}$, but they are quite contradictory (here we just recall the extensive discussion on the slope anomalies $[13,14]$ ) since they have been derived mostly from angular distributions taken at energies above the Coulomb barrier. In this situation the values of $P_{t r}$ at large distances $D$ are coming from forward scattering angles where processes other than the direct one may contribute to the cross section, as it is clearly seen from the $Q$-value spectra [15]. The coming into operation of large acceptance magnetic spectrometers made it possible to perform measurements on multinucleon transfer reactions with good ion identification, also at very low energies. With this experimental advance we are now in a position to extend to more cases the formalism outlined in Refs. [10,11] and thus to provide an answer on the origin of the large enhancement factors reported for these reactions (cf. Refs. [16-18]).

In this work we present the results of a sub-barrier transfer measurement performed for the ${ }^{40} \mathrm{Ca}+{ }^{96} \mathrm{Zr}$ system in inverse kinematics with the PRISMA spectrometer [19,20]. Projectile and target are closed-shell nuclei (or nearly so) for both neutrons and protons, thus providing suitable conditions for a proper study of the mechanism that governs the exchange of nucleons in a transfer process [21]. After a description of the employed experimental techniques we present the experimental results for neutron transfer channels. Data are then compared with calculations performed within the semiclassical framework.

\section{THE EXPERIMENT}

In direct kinematics the transfer probability at large distances corresponds to fragments that are scattered at forward angles. In this angular range the overwhelming yield of elastically scattered particles often prevents a good identification for isotopes close to the projectile. At energies below the barrier measurements of heavy-ion-transfer reaction products present other significant technical difficulties. Backscattered ions have low kinetic energies which severely limit their identification, making available data extremely scarce. To partly overcome these problems, two methods have been so far employed, one that identifies the (heavy) target recoils at zero degrees with recoil mass spectrometers [22,23], and a second where one makes use of inverse kinematics to detect the lighter targetlike particles with a magnetic spectrometer at forward angles $[24,25]$. Here the energy of the recoiled light fragment is quite large; thus one can obtain a good resolution in mass and nuclear charge.

In the data so far obtained at sub-Coulomb energies, the small solid angle of conventional spectrometers limited the statistical accuracy. Here we made use of the large solid angle spectrometer PRISMA. By employing inverse kinematics and by detecting ions at very forward angles, we have, at the same time, enough kinetic energy of the outgoing recoils (for energy and therefore mass resolution) and forward-focused angular distribution (high efficiency).

We measured an excitation function with a ${ }^{96} \mathrm{Zr}$ beam delivered from the XTU-Tandem + ALPI superconducting booster of LNL with average currents of $\sim 1$ pnA onto a $50 \mu \mathrm{g} / \mathrm{cm}^{2}{ }^{40} \mathrm{Ca}$ target. The target was made of a $\mathrm{CaF}_{2}$ material, strip ( $2 \mathrm{~mm}$ width), supported on a $15 \mu \mathrm{g} / \mathrm{cm}^{2} \mathrm{C}$ backing. The target isotopic enrichment was $\sim 99.98 \% .{ }^{42} \mathrm{Ca}$ impurities were at the level of $10^{-5}$ compared to ${ }^{40} \mathrm{Ca}$. The bombarding energy of ALPI has been varied in steps of $15 \mathrm{MeV}$ from 330 to $275 \mathrm{MeV}$. The lowest energy $E_{\text {lab }}=$ $255 \mathrm{MeV}$ was measured with the Tandem only, to have a precise reference (energy accuracy is better than $0.1 \%$ ). For each ALPI energy, measurements were performed at two additional energies by placing in front of the $\mathrm{Ca}$ targets one and two $\mathrm{C}$ foils, with a thickness of $85 \mu \mathrm{g} / \mathrm{cm}^{2}$ each, in such a way to degrade the ${ }^{9} \mathrm{Zr}$ beam by about 6 and $12 \mathrm{MeV}$, respectively. This procedure has been adopted to decrease the beam time loss due to the tuning of the ALPI cavities at each change of energy. So a complete excitation function has been measured from the Coulomb barrier to $\simeq 25 \%$ below, measuring the transfer yields down to $\sim 15.5 \mathrm{fm}$ of distance of closest approach.

Absolute normalization of cross sections and normalizations between different runs was ensured by two silicon surface barrier monitor detectors placed at $\theta_{\mathrm{lab}}=55^{\circ}$ and $\theta_{\mathrm{lab}}=60^{\circ}$ with respect to beam direction and at a distance of $\sim 40 \mathrm{~cm}$ from the target. The monitors detected the Rutherford-scattered Ca-like (as well as ${ }^{19} \mathrm{~F}$ and ${ }^{12} \mathrm{C}$ ) recoils. The energy of the ${ }^{96} \mathrm{Zr}$ beam delivered by the ALPI booster was defined with a 


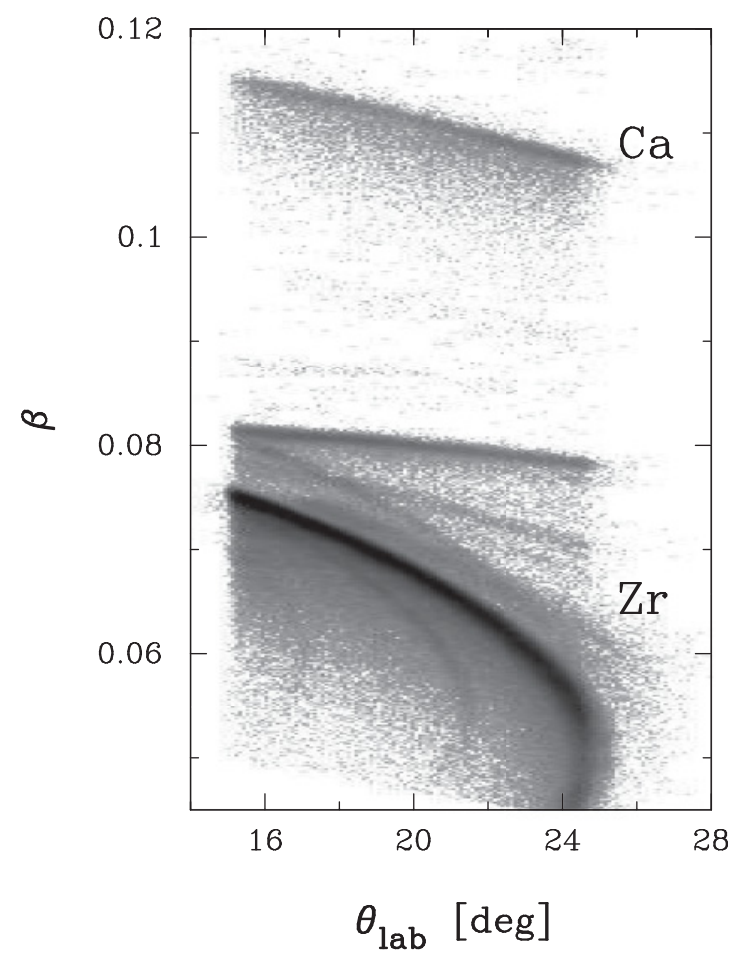

FIG. 1. Velocity $(\beta=v / c)$ vs in-plane scattering angle $\left(\theta_{\mathrm{lab}}\right)$ for the reaction ${ }^{96} \mathrm{Zr}+{ }^{40} \mathrm{Ca}$ at $E_{\mathrm{lab}}=330 \mathrm{MeV}$. The magnetic fields of the spectrometer were set to bring the $\mathrm{Ca}$ ions with maximum yield near the center of the focal plane area. The events at large $\beta$ correspond to $\mathrm{Ca}$-like recoils, and those at lower $\beta$ to $\mathrm{Zr}$-like ions scattered from $\mathrm{Ca}$, as well as from other elements (see text for details).

precision $\sim 2 \%$. The centroids of the peaks of the Rutherfordscattered targetlike $\mathrm{Ca}, \mathrm{F}$, and $\mathrm{C}$ ions in the two monitor detectors have been taken into account via a minimization procedure with the constraint to pass through the points at $E_{\text {lab }}=255 \mathrm{MeV}$ measured with the Tandem only, defining the final energies better than $1 \%$.

Ca-like recoils have been detected by PRISMA at $\theta_{\text {lab }}=$ $20^{\circ}$, corresponding to $\theta_{\text {c.m. }} \simeq 140^{\circ}$. In Fig. 1 we show an example of two-dimensional plot velocity (reconstructed via time-of-flight) vs in-plane scattering angle. One sees the events corresponding to $\mathrm{Ca}$-like recoils as well as those corresponding to $\mathrm{Zr}$-like ions entering into PRISMA. The ridges of these $\mathrm{Zr}$-like ions are due to scattering on $\mathrm{Ca}$ (main ridge presenting a bending around the kinematic limiting angle $\theta_{\mathrm{lab}}=24.6^{\circ}$ ), on lighter elements (with smaller limiting angles), and on other heavier elements. The almost horizontal ridge, with high yield and with a $\beta \sim 0.08$, is due to $\mathrm{Zr}$ scattering in direct kinematics. Full identification in mass $(A)$, nuclear charge $(Z)$, and $Q$ value of transfer reaction products has been achieved. The nuclear charge $Z$ has been obtained through the measurement of energy loss $\Delta E$ and total energy $E$ in the ionization chamber located at the focal plane. In Fig. 2 we present an example of a $\Delta E-E$ matrix integrated over the whole angular acceptance of the spectrometer, where the $Z$ selectivity in the $\mathrm{Ca}$ region is shown. In the same figure one also sees the tail of the Zr-like events. Mass identification has been based on an event-by-event reconstruction of the ion trajectory inside the

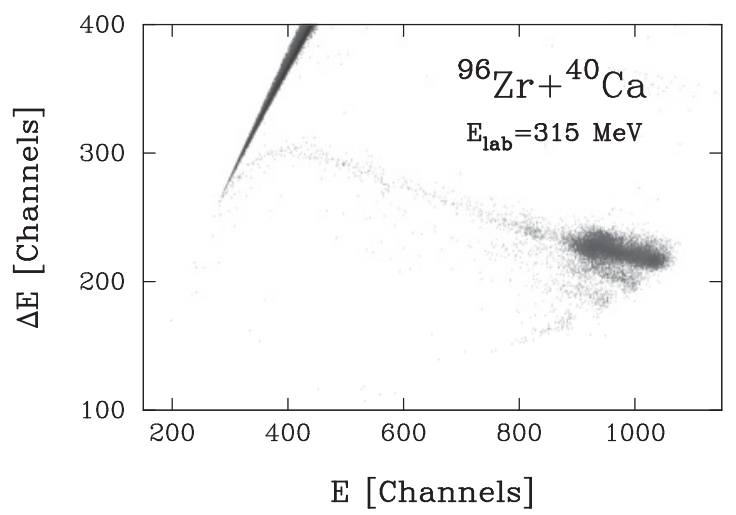

FIG. 2. $\Delta E-E$ matrix obtained at the indicated bombarding energy and with the spectrometer at $\theta_{\mathrm{lab}}=20^{\circ}$. The most intense spot corresponds to $Z=20$ (Ca isotopes), the lower ones to $Z=19$ and $Z=18$, respectively. The tail of events corresponding to $Z$ r-like ions entering into the spectrometer are also visible.

magnetic elements, using two-dimensional entrance and exit positions and time of flight [20]. Since the yields for proton transfer drop off very rapidly at these sub-barrier energies, in this work we discuss only pure neutron transfer channels where sufficient statistics have been accumulated. Mass spectra at representative energies are shown in Fig. 3, where a clear separation of different isotopes is visible. At energies close to the Coulomb barrier we observe the population of more than four neutron pick-up channels, while at sub-barrier energies only one or at most two neutron transfers survive. A significant transfer yield could be detected at the level of $10^{-4}$ with respect to the elastic channel.

The total kinetic energy loss (TKEL) distributions are shown in Fig. 4 for the quasielastic (elastic + inelastic) and the $+1 n$ and $+2 n$ transfer channels at three different bombarding energies. They are constructed from the measurement of the vector velocity of the selected ions on the assumption of binary kinematics. The width of the quasielastic channel is $\sim 3 \mathrm{MeV}$, i.e., compatible with the overall resolution of PRISMA and beam-energy indetermination. The ground-state $Q$ values, indicated with vertical dotted lines, are at $+0.51 \mathrm{MeV}$ for the $+1 n$ channel and at $+5.53 \mathrm{MeV}$ for the $+2 n$ channel. The spectra for the $+1 n$ channel have a maximum that slightly moves toward higher energy losses with the increase of the bombarding energy, in accordance with the energy dependence of the optimum $Q$ value, that for neutrons is very close to zero. The width is constant below the barrier and grows above it, reflecting the opening of other reaction channels. The spectra of the $+2 n$ display a similar behavior but, in this case, the reaction mechanism leaves unpopulated the ground-state transition where most of the pairing strength should be concentrated.

\section{A. Transfer probabilities}

The differential cross sections are obtained by taking into account the geometry of monitor and PRISMA detectors and assuming an energy and mass independent ion transmission through the spectrometer. This assumption is well justified 


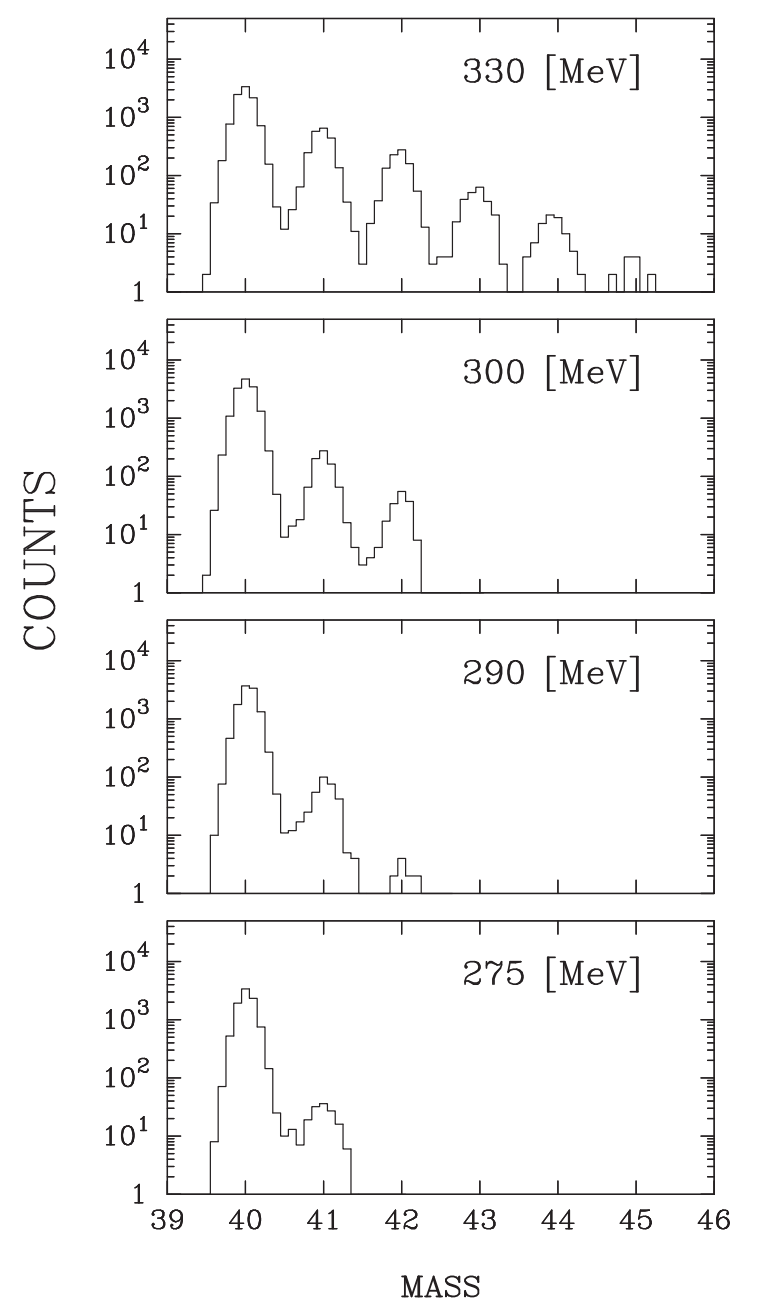

FIG. 3. Mass distributions for pure neutron transfer channels obtained in the reaction ${ }^{96} \mathrm{Zr}+{ }^{40} \mathrm{Ca}$ at the indicated bombarding energies of ${ }^{96} \mathrm{Zr}$. Ca-like recoils have been detected with the spectrometer at $\theta_{\text {lab }}=20^{\circ}$. The obtained resolution is $\Delta A / A \sim 1 / 150$, which allows clear separation of the different isotopes.

in the present experiment, as we are dealing with pure neutron transfer channels which, at sub-Coulomb energies, have narrow $Q$-value distributions weakly dependent on the isotope and well within the acceptance of the spectrometer. Exploiting the fact that the angular distributions at all measured energies have the same shape, in order to maximize the statistical accuracy we integrated over the full angular range of the spectrometer and ascribed the corresponding cross section and transfer probability $P_{t r}$ to the central angle that is then used to derive the distance of closest approach [see Eq. (2)]. To get the absolute normalization, we used the fact that the elastically scattered ${ }^{40} \mathrm{Ca}$ ions in PRISMA at energies well below the barrier correspond to pure Rutherford events.

The extracted transfer probabilities for the $+1 n,+2 n$, and $+3 n$ transfer channels are shown in Fig. 5 as a function of the distance of closest approach $D$. In the same figure are also shown the results for the $+4 n$ channel, where data could be extracted only at the highest energies.
The transfer probabilities are very well described by an exponential function with a decay length that gets smaller as the number of transferred neutrons increases. This behavior of the transfer probabilities suggests a simple phenomenological interpretation of the data. The full line represents a fit to the transfer probability for the $+1 n$ channel. By using a simple transfer model based on the exchange of independent particles that predicts for the two-particle channel a probability proportional to the square of the single-particle probability, one can obtain a nice description for the $+2 n$ and $+3 n$ channels with the equations $P_{2 n}=3\left(P_{1 n}\right)^{2}$ and $P_{3 n}=3\left(P_{1 n}\right)^{3}$. These are shown with dashed lines in the same figure. The factor 3 appearing in the previous expressions represents an enhancement factor whose origin is addressed in the following section.

\section{RESULTS AND DISCUSSION}

In this section we shortly outline the formalism we use for the analysis of one- and two-nucleon transfer data. The transfer channels, although many, are quite weak so as to allow a perturbative approach. In first order the semiclassical amplitude for a transition from the entrance channel $\left|\psi_{\alpha}\right\rangle$ to the final channel $\left|\psi_{\beta}\right\rangle$ is given by

$$
c_{\beta}(\ell)=\frac{1}{i \hbar} \int_{-\infty}^{+\infty}\left\langle\psi_{\beta}\left|\left(V_{\alpha}-U_{\alpha}\right)\right| \psi_{\alpha}\right\rangle_{\mathcal{R}} e^{i\left(E_{\beta}-E_{\alpha}\right) t / \hbar} d t
$$

where the time integral has to be performed along the classical trajectory of partial wave $\ell$. $V_{\alpha}$ represents the interaction among all the nucleons in the entrance channel mass partition and $U_{\alpha}$ its expectation value in their ground-state configurations, usually identified with the real part of the ion-ion optical potential. With $\mathcal{R}$ we indicated the distance between the center-of-mass of the two nuclei, which in the semiclassical approximation is defined as the average between the distances in the entrance and exit channel. The channel wave function of energy $E_{\beta}$,

$$
\left|\psi_{\beta}(t)\right\rangle=\psi_{b}\left(\xi_{b}, t\right) \psi_{B}\left(\xi_{B}, t\right) e^{-\frac{i}{\hbar} \delta_{\beta}(t)},
$$

is defined as the product of the two intrinsic wave functions times a phase factor $\delta_{\beta}(t)$ that takes into account that the two ions are moving along a classical trajectory developing in a nuclear plus Coulomb field. The $\xi_{b}$ and $\xi_{B}$ represent the spin and space coordinates of the nucleons in projectilelike and targetlike systems, respectively.

Considering the stripping of a single nucleon $a(b+1)+$ $A \rightarrow b+B(A+1)$, for even-even nuclei $\left(I_{a}=I_{A}=0\right)$, the first-order amplitude for the transition of a nucleon from the single-particle state $a_{1}^{\prime} \equiv\left(n_{1}^{\prime}, l_{1}^{\prime}, j_{1}^{\prime}\right)$ in the projectile to the single-particle state $a_{1} \equiv\left(n_{1}, l_{1}, j_{1}\right)$ in the target may be written in the form

$$
c_{\beta}(\ell)=-i \sum_{\lambda \mu} \frac{(-1)^{\lambda+\mu}}{\sqrt{2 \lambda+1}}\left\langle j_{1}^{\prime} m_{1}^{\prime} \lambda-\mu \mid j_{1} m_{1}\right\rangle I_{\lambda \mu}^{\left(a_{1}, a_{1}^{\prime}\right)}(\ell),
$$

where $\lambda$ is the transferred angular momentum, $\mu$ its third component, and $I_{\lambda \mu}^{\left(a_{1}, a_{1}^{\prime}\right)}$ is the orbital integral of the radial form 

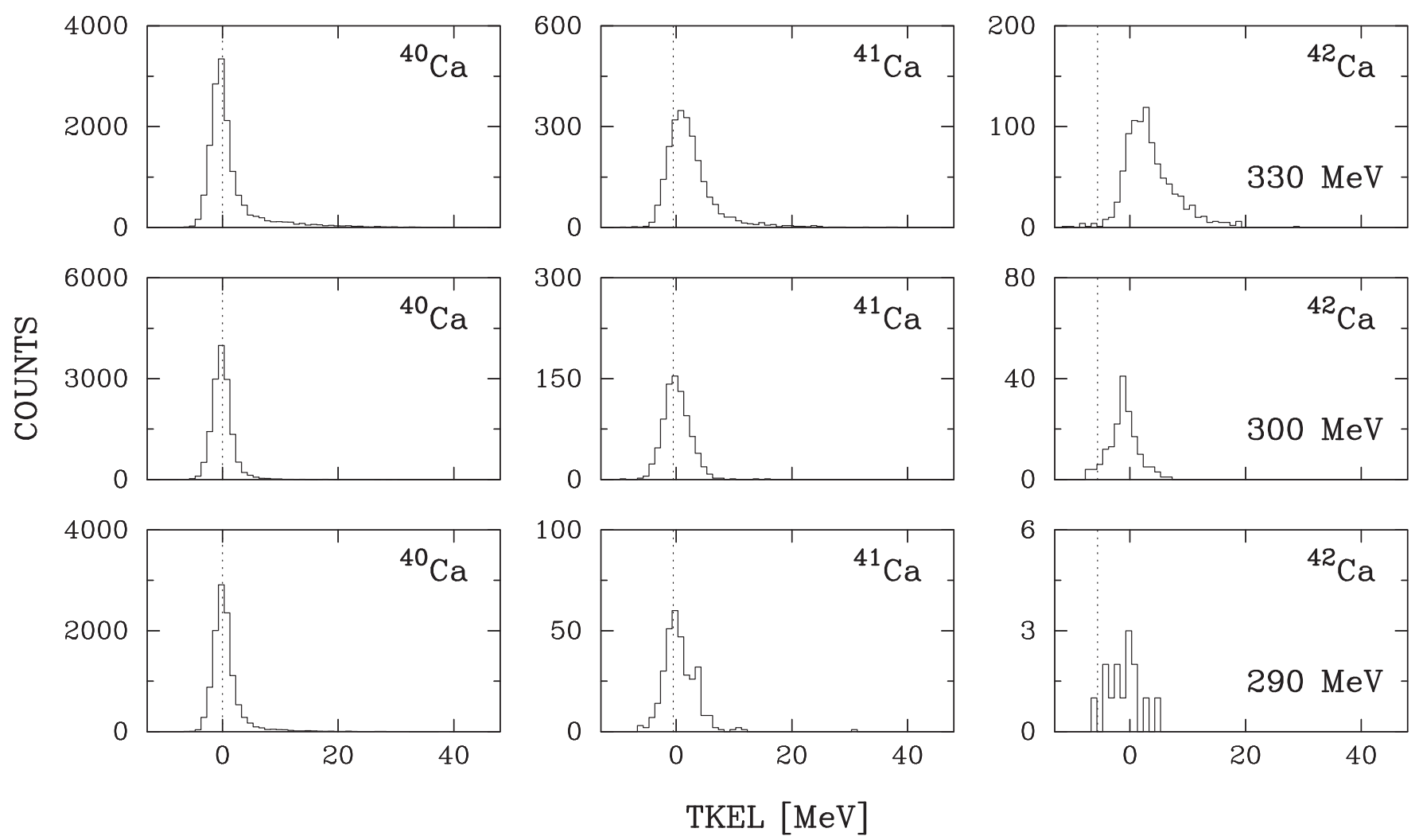

FIG. 4. Experimental TKEL distributions for the elastic(+inelastic) and $+1 n$ and $+2 n$ transfer channels obtained in the reaction ${ }^{96} \mathrm{Zr}+{ }^{40} \mathrm{Ca}$ at the indicated bombarding energies. The vertical dotted lines represent the ground-state $Q$ values.

factor, which is calculated to be

$$
\begin{aligned}
I_{\lambda \mu}^{\left(a_{1}, a_{1}^{\prime}\right)}(\ell)= & \sqrt{\frac{2 \pi a_{t r}}{\ddot{r}_{o} \hbar^{2}}} Y_{\lambda \mu}(\pi / 2,0) f_{\lambda 0}\left(D_{\ell}\right) \\
& \times e^{-\frac{a_{t r}}{2 r_{0} \hbar^{2}}\left(\Delta E-Q_{o p t}-\hbar \mu \dot{\phi}(0)\right)^{2}} .
\end{aligned}
$$

With $f_{\lambda 0}$ we have indicated the radial form factor of multipolarity $\lambda$ (as a function of the distance of closest approach $D_{\ell}$ ), with $\Delta E$ the $Q$ value of the transition, with $Q_{\text {opt }}$ the optimum $Q$ value, while $\ddot{r}_{0}$ and $\dot{\phi}(0)$ are the radial acceleration and the angular velocity at the distance of closest approach. The transfer probability is readily calculated from the amplitude as

$$
\begin{aligned}
P_{\beta}(\ell)=P_{\left(a_{1}, a_{1}^{\prime}\right)}(\ell)= & \sum_{m_{1}^{\prime}, m_{1}}\left|c_{\beta}(\ell)\right|^{2} \\
= & U^{2}\left(a_{1}, I_{A}\right) V^{2}\left(a_{1}^{\prime}, I_{a}\right) \sum_{\lambda \mu} \frac{2 j_{1}^{\prime}+1}{2 \lambda+1} \\
& \times\left|I_{\lambda \mu}^{\left(a_{1}, a_{1}^{\prime}\right)}(\ell)\right|^{2},
\end{aligned}
$$

where we wrote explicitly the occupation probabilities for the single-particle states $a_{1}^{\prime}$ and $a_{1}$ in projectile and target, respectively.

In deriving the expression (6) we exploited the fact that the radial form factor, in the tail region, may be well approximated with an exponential tail,

$$
f_{\lambda 0}(\mathcal{R}) \sim e^{-\left(\mathcal{R}-R_{0}\right) / a_{t r}}
$$

and that the trajectory of relative motion may be approximated with a parabola osculating the true trajectory at the distance of closest approach $D_{\ell}$. The optimum $Q$ value takes into account that the amplitude is maximal for transitions that have a good matching between the entrance and exit channel trajectories. Its expression may be found in Ref. [9]. The actual width of the experimental TKEL spectra (see Fig. 4) derives from the distribution of the form-factor strength weighted by the adiabatic cut-off function [Eq. (6)].

The parameter $a_{t r}$ defines the range of the transfer form factor. Its value is related to the binding energy of the transferred nucleon in the target system if one adopts the so-called prior representation, while is related to the binding energy in the projectile if one adopts the post representation. These different representations, which derive from the nonorthogonality of the channel wave functions, is of minor importance for reactions involving nuclei in the $\beta$-stability valley but may lead to conflicting arguments when dealing with exotic beams where the Fermi energies of the projectile and target are very different. These considerations also have some relevance here, since we chose to represent the transfer probabilities as a function of the distance of closest approach $\left(D_{\ell}\right)$ in order to have a direct visualization of the tail of the transfer form factor. From Eqs. (5)-(7) we see that the probability for a given transfer is proportional to the square of the form factor times a term (adiabatic cut-off function) that depends on the optimum $Q$ value of the reaction. This result clearly shows that it is convenient to extract from the data the transfer probability $P_{t r}(D)$ and to plot it as a function 


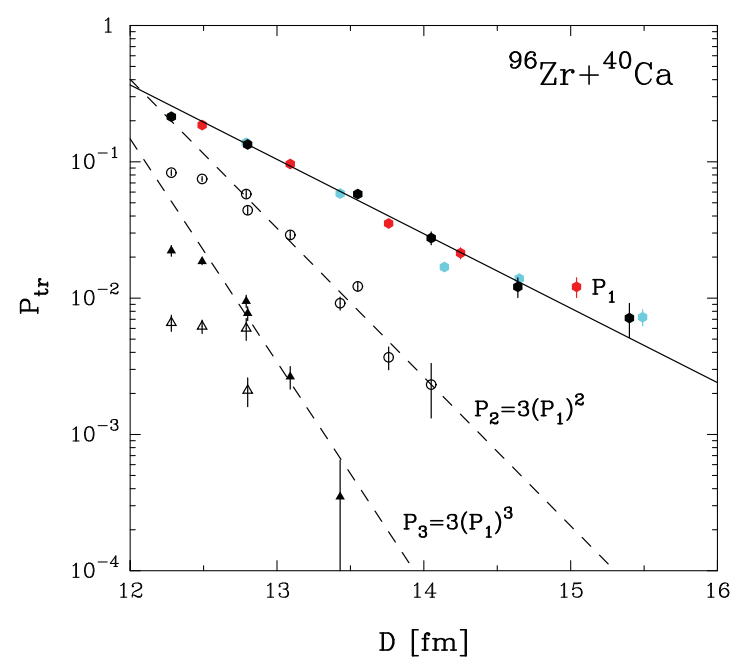

FIG. 5. (Color online) Transfer probabilities as a function of the distance of closest approach for neutron transfer channels. Points are the experimental data: $+1 n$ (full circles), $+2 n$ (empty circles), $+3 n$ (full triangles), and $+4 n$ (empty triangles). For the $+1 n$ channel we indicated in black the data obtained from the nominal bombarding energy. The energies, in the center of the target, are 329.2, 315.9, $298.4,287.7,276.1$, and $262.6 \mathrm{MeV}$, corresponding to the distances of closest approach 12.28, 12.80, 13.55, 14.05, 14.64, and $15.40 \mathrm{fm}$. With red and cyan colors we indicated the data obtained with one and two degraders, respectively. Quoted errors are statistical only, errors on $D$, reflecting energy indetermination, are $\leqslant 1 \%$. The full line is a fit according to the expression $P_{1 n}=\exp (13.95-1.25 D)$. In the fit we excluded the points at the highest measured energies where absorption starts to be a significant fraction of the elastic channel. The dashed lines correspond to the shown equations.

of the distance of closest approach. However, one has to keep in mind that the transfer probability extracted from inclusive cross section will have a tail representing the binding energies of the transferred nucleon only if the width of the $Q$-value spectra do not depend strongly on the bombarding energy.

Our treatment of the two-particle transfer process follows very closely the one of Ref. [12], so here we simply remind some initial considerations referring to the above references for details. To calculate the amplitude for the transfer of two nucleons one has to solve the well-known system of semiclassical coupled equations up to the second-order Born approximation. This may be written in the form

$$
c_{\beta}(\ell)=\left(c_{\beta}\right)_{(1)}+\left(c_{\beta}\right)_{\text {ort }}+\left(c_{\beta}\right)_{\text {succ }},
$$

where the first term $\left(c_{\beta}\right)_{(1)}$ describes the simultaneous transfer of the pair of nucleons, the second term arises from the nonorthogonality of the channel vectors $\psi_{\gamma}$, and the last term describes the successive process via an intermediate channel $\gamma$ :

$$
\begin{aligned}
& \left(c_{\beta}\right)_{\text {succ }} \\
& =\left(\frac{1}{i \hbar}\right)^{2} \sum_{\gamma} \int_{-\infty}^{+\infty} d t<\psi_{\beta}\left|\left(V_{\gamma}-U_{\gamma}\right)\right| \psi_{\gamma}>_{\mathcal{R}} \\
& \quad \times e^{i\left(E_{\beta}-E_{\gamma}\right) t / \hbar} \\
& \quad \int_{-\infty}^{t} d t^{\prime}<\psi_{\gamma}\left|\left(V_{\alpha}-U_{\alpha}\right)\right| \psi_{\alpha}>_{\mathcal{R}} e^{i\left(E_{\gamma}-E_{\alpha}\right) t / \hbar},
\end{aligned}
$$

where for the initial $(\alpha)$, intermediate $(\gamma)$, and final $(\beta)$ channels we have made the following identification (we consider a stripping reaction):

$$
a(b+2)+A \rightarrow c(b+1)+C \rightarrow b+B .
$$

It is very easy to demonstrate that in an independent particle description the first and second term cancel exactly (one can in fact resort to the completeness relation). Although in actual cases this does not fully hold, since the single-particle energies of the intermediate states cannot be kept the same in the two-step process, it was shown in many works [10-12] that the cancellation almost persists, so we here consider that

$$
\left(c_{\beta}\right)_{(1)}=\left(c_{\beta}\right)_{\mathrm{ort}}
$$

and we use only the successive term. By using the two-particle parentage expansion of the projectile $(a)$ and of the target-like $(B)$ wave functions one can express the successive amplitude in terms of the one-particle transfer form factor introduced above for the calculation of one-nucleon transfer reactions. Working in the $m$ representation one can write (cf. Ref. [12]):

$$
\begin{aligned}
\left(c_{\beta}\right)_{\mathrm{succ}}= & \frac{1}{\hbar^{2}} \sum_{a_{1}, a_{1}^{\prime}} B^{(A)}\left(a_{1} a_{1} ; 0\right) B^{(a)}\left(a_{1}^{\prime} a_{1}^{\prime} ; 0\right) 2 \frac{(-1)^{j_{1}+j_{1}^{\prime}}}{\sqrt{\left(2 j_{1}+1\right)} \sqrt{\left(2 j_{1}^{\prime}+1\right)}} \sum_{m_{1} m_{1}^{\prime}}(-1)^{m_{1}+m_{1}^{\prime}} \\
& \times \int_{-\infty}^{+\infty} d t f_{m_{1} m_{1}^{\prime}}(\mathcal{R}) e^{i\left[\left(E_{\beta}-E_{\gamma}\right) t+\delta_{\beta \gamma}(t)+\hbar\left(m_{1}^{\prime}-m_{1}\right) \Phi(t)\right] / \hbar} \\
& \times \int_{-\infty}^{t} d t f_{-m_{1}-m_{1}^{\prime}}(\mathcal{R}) e^{i\left[\left(E_{\gamma}-E_{\alpha}\right) t+\delta_{\gamma \alpha}(t)-\hbar\left(m_{1}^{\prime}-m_{1}\right) \Phi(t)\right] / \hbar}
\end{aligned}
$$

The two-dimensional time integral is easily calculated in terms of the one-dimensional time integral encountered in the calculation of the one-neutron transfer amplitude by using the step function $\theta\left(t-t^{\prime}\right)$ to complete the second time integral up to $+\infty$ :

$$
\theta\left(t-t^{\prime}\right)=\frac{1}{2}\left[1+\epsilon\left(t-t^{\prime}\right)\right]=\frac{1}{2}\left[1+\frac{i}{\pi} \mathcal{P} \int_{-\infty}^{+\infty} \frac{d q}{q} e^{i q(t-t)}\right] .
$$




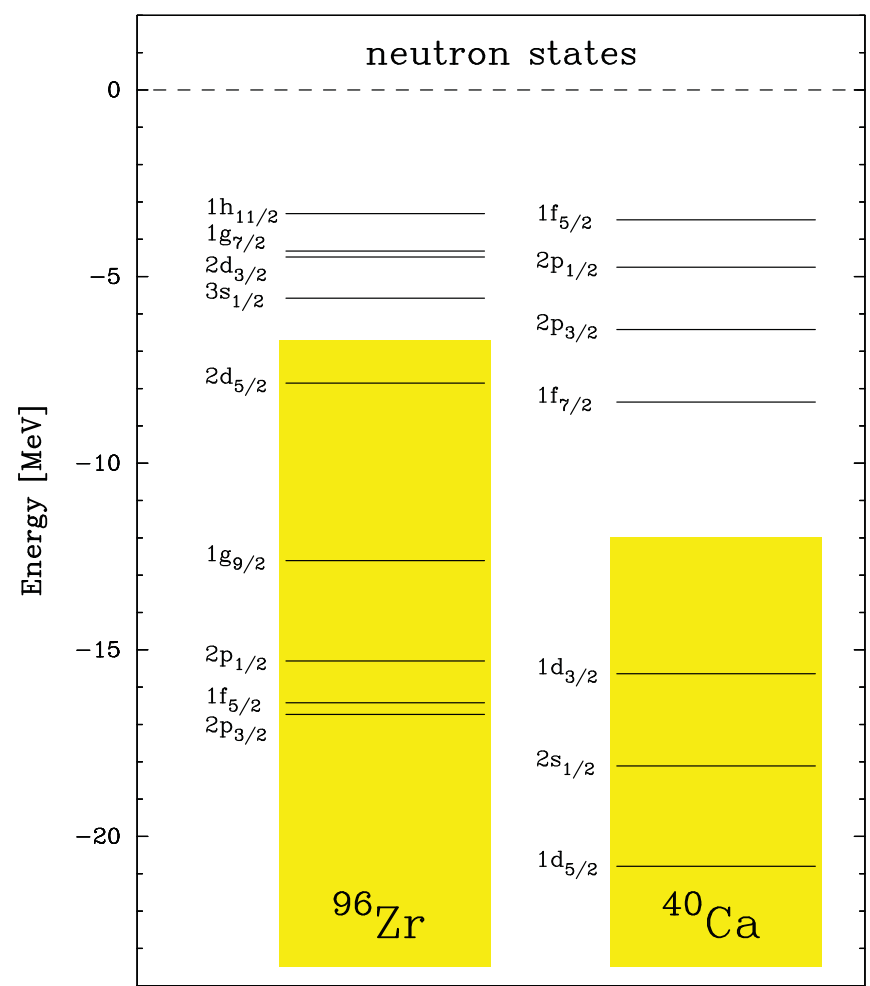

FIG. 6. (Color online) Neutron single-particle levels in ${ }^{96} \mathrm{Zr}$ and ${ }^{40} \mathrm{Ca}$.

For more details see, for example, Ref. [12].

The $B$ coefficients appearing in the above expression are the spectroscopic factors for two particles. For superfluid systems described in the BCS approximation they may be expressed as a function of the occupation probabilities $U$ and $V$ as

$$
B^{(A)}\left(a_{1} a_{1} ; 0\right)=(-1)^{l_{1}} \sqrt{j_{1}+1 / 2} U\left(a_{1} ; 0\right) V\left(a_{1} ; 0\right),
$$

while for pairing vibrational systems, described in the TammDancoff approximation, they are given by

$$
B^{(A)}\left(a_{1} a_{1} ; 0\right)=X^{(A)}\left(a_{1}\right) .
$$

In applying the above formalism in the calculation of inclusive cross sections, we cannot just add-up the probabilities of all possible transitions - the number of transitions may be very large and the sum may exceed one, even if the single transitions are quite small. The inclusive transfer probability $P_{\text {inc }}$ has to be calculated as

$$
P_{\mathrm{inc}}=\sum_{\beta} P_{\beta \alpha} \prod_{\gamma \neq \beta}\left(1-P_{\gamma \alpha}\right) \simeq e^{-\sum_{\gamma} P_{\gamma \alpha}} \sum_{\beta} P_{\beta \alpha},
$$

where the sum has to be extended over all open channels. The first term of the right-hand side of the last equation

$$
P_{o}=e^{-\sum_{\gamma} P_{\gamma \alpha}}
$$

provides the probability to remain in the elastic channel. It is from this equation that the imaginary part of the optical potential has been calculated [26,27].

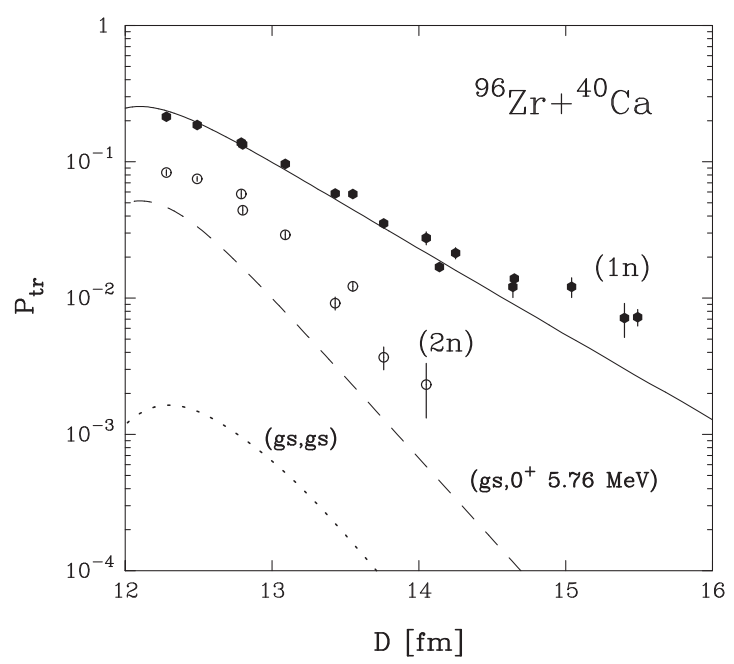

FIG. 7. Theoretical transfer probabilities for one- and twoparticle transfer (lines) in comparison with the experimental data (points). The full line represents the inclusive transfer probability for one-neutron transfer, the dotted line the ground-ground state transition for the two-neutron transfer, and the dashed line the transition to the first $0^{+}$excited state at $5.76 \mathrm{MeV}$ in ${ }^{42} \mathrm{Ca}$ (see text).

\section{A. One-neutron transfer}

To compute the inclusive one-neutron stripping cross section (remember that we are working in inverse kinematic so that ${ }^{96} \mathrm{Zr}$ is the projectile) we use Eq. (7) to calculate the transfer probability for a given single-particle transition and we obtain the total transfer probability by summing over all possible transitions that can be constructed from the single-particle states in the projectile and target shown in Fig. 6. The set of single-particle states covers a full shell below the Fermi level for the ${ }^{96} \mathrm{Zr}$ and a full shell above for the ${ }^{40} \mathrm{Ca}$. The spectroscopic factors have been all set to one. In fact, when dealing with inclusive cross sections we can neglect the fragmentation of the single-particle strength over several states and suppose that the full strength is concentrated on a single transition. The calculated total transfer probability is shown in Fig. 7 with a full line.

To calculate the single-particle transfer form factors we have generated the wave functions of the single-particle states by using a shell model potential of standard Woods-Saxon form with $a=0.65 \mathrm{fm}$ and $r_{0}=1.25 \mathrm{fm}$. Its depth has been adjusted so as to reproduce the experimental binding energy. The same shell model is then used as the interaction responsible for the transfer. For the ion-ion potential entering in the calculation of the form factor we use the empirical potential of Ref. [9]. The data are quite well described both in magnitude and slope, and this is not unexpected since the one-neutron transfer form factors are constructed by using the experimental single-particle energies.

\section{B. Two-neutron transfer}

The formalism discussed above for the transfer of two neutrons may only be applied to the transfer of a $J=0^{+}$ 
TABLE I. Wave functions for the $0^{+}$ground state of ${ }^{94} \mathrm{Zr}$ and for the $0^{+}$ground state of ${ }^{42} \mathrm{Ca}$ with its first $0^{+}$excited state at $5.76 \mathrm{MeV}$ (see text for more details).

\begin{tabular}{lccccc}
\hline \hline \multicolumn{2}{c}{${ }^{94} \mathrm{Zr}$} & & ${ }^{42} \mathrm{Ca}$ & \\
\hline$a_{i}$ & B.E. $(\mathrm{MeV})$ & $B\left(a_{i}, a_{i} ; 0^{+}\right)$ & $a_{i}$ & B.E. $(\mathrm{MeV})$ & $B\left(a_{i}, a_{i} ; 0^{+}\right)$ \\
\hline $2 d_{3 / 2}$ & -16.730 & -0.0579 & & \\
$1 f_{5 / 2}$ & -16.420 & -0.0734 & & & \\
$2 p_{1 / 2}$ & -15.300 & -0.0485 & & & \\
$1 g_{9 / 2}$ & -12.610 & 0.1663 & & & \\
$2 d_{5 / 2}$ & -7.854 & 0.8360 & $\left.1 a_{i}, a_{i} ; 0_{2}^{+}\right)$ & \\
$3 s_{1 / 2}$ & -5.579 & 0.1693 & $2 p_{3 / 2}$ & -8.620 & 0.93 \\
$2 d_{3 / 2}$ & -4.476 & 0.1617 & $2 p_{1 / 2}$ & -4.760 & -0.93 \\
$1 g_{7 / 2}$ & -4.315 & 0.2181 & $1 f_{5 / 2}$ & -3.380 & 0.19 \\
$1 h_{11 / 2}$ & -3.314 & 0.2075 & & 0.21 \\
\hline \hline
\end{tabular}

pair. The ground-state wave function for the ${ }^{94} \mathrm{Zr}$ is obtained from a BCS calculation by adopting the single-particle states of Fig. 6 and a state-independent pairing interaction $G=0.218$ that gives a pairing gap $\Delta=0.746 \mathrm{MeV}$ with a Fermi energy of $7.6523 \mathrm{MeV}$. The calculated $B\left(a_{i} a_{i} ; 0\right)$ coefficients are reported in Table I.

For the description of ${ }^{42} \mathrm{Ca}$ we diagonalize the Hamiltonian $H=H_{0}+V_{\text {res }}$, where $H_{0}$ is the single-particle Hamiltonian whose eigenvalues are the single-particle states shown in Fig. 6 (only the states above the Fermi energy of ${ }^{40} \mathrm{Ca}$ are considered), and $\mathrm{V}_{\text {res }}$ is a surface-delta two-body interaction simplified to have constant radial matrix elements. The explicit expression for the matrix element can be found in Ref. [28] (cf. page 32). The model space contains only a two-particle configuration coupled to $0^{+}$. The strength of the interaction is tuned so that the lowest eigenstate of the Hamiltonian reproduces the separation energy between ${ }^{42} \mathrm{Ca}$ and ${ }^{40} \mathrm{Ca}(-19.843 \mathrm{MeV})$. The resulting $0^{+}$states are at energies of 5.76,9.10, and $11.96 \mathrm{MeV}$. The $B\left(a_{i} a_{i} ; 0\right)$ coefficients for the ground state and the $0_{2}^{+}$ state at $5.76 \mathrm{MeV}$ are shown in Table I. Since the model space for the description of ${ }^{42} \mathrm{Ca}$ is very small, the model does not reproduce all the experimental $0^{+}$states. The ones at 1.84 and 3.30 MeV are recognized to derive from core excitations and are outside our model space. We point out that the core-excited states should be very weakly populated in a transfer reaction, since they have to rely on higher order processes like inelastic excitation followed by transfer or vice versa.

In Fig. 7 with a dotted line we show the calculated probability for the ground-ground state transition. Clearly, this transition does not contribute to the total transfer strength, in agreement with what was pointed out in the discussion of the $Q$-value spectra. In the same figure, with a dashed line, we show the predicted transfer probability for the transition to the $0^{+}$state at $5.76 \mathrm{MeV}$ in ${ }^{42} \mathrm{Ca}$.

It is apparent that the contribution of this transition is much larger than the ground-state contribution. Besides its good match with the optimum $Q$ value, this is mostly due to the $2 p_{3 / 2}$ component that dominates its wave function and that has a much larger form factor. The centrifugal barrier for an $f$ state is in fact larger than the one for a $p$ state. The contributions of the other high-energy $0^{+}$states, at 9.10 and $11.96 \mathrm{MeV}$, are negligible and are not shown. Note that $0^{+}$states in the energy region of $\sim 6 \mathrm{MeV}$ were strongly populated in $(t, p)$ reactions [29].

To try to come to an understanding of the still-missing factor for the description of the total transfer cross section, we recall that the data are inclusive and that our calculation includes only the transfer to $0^{+}$states. From a previously performed measurement of the same reaction at a energy higher than those of the present work [20], where $\gamma$ rays were measured in coincidence with the reaction fragments, it has been possible to reconstruct, for ${ }^{42} \mathrm{Ca}$ and ${ }^{94} \mathrm{Zr}$ nuclei, the level schemes shown in Fig. 8 together with the estimated strength (see caption). From these spectra one sees that in the reaction are populated states with relatively high angular momentum and states with non-natural parity (we recall that $I_{a}=0$ and $I_{A}=0$ ). From an analysis of the strength distribution, the population of these states turns out to account for $\sim 75 \%$ of the total yield. Thus it seems plausible to ascribe the enhancement factor of $\sim 3$ to the fact that the two-nucleon transfer reaction does not populate only $0^{+}$states but it is much richer, so that more complicated two-particle correlations have to be taken into account. To this purpose we stress, in particular, the population of the non-natural parity states, since their population indicates that in the $2 n$-transfer reaction the effect of the recoil is important. We also stress the role played by the single-particle states $f_{7 / 2}$ and $p_{3 / 2}$. While the first dominates the ground-state wave function of ${ }^{42} \mathrm{Ca}$, the second dominates the wave function of the $0^{+}$state at $5.76 \mathrm{MeV}$, the $p_{3 / 2}$ state providing the major contribution to the transfer process.

\section{SUMMARY}

An excitation function for multinucleon transfer channels has been measured in a broad energy range and far below the Coulomb barrier. Measurements have been done in inverse kinematics, detecting the lighter targetlike ions with the large solid angle magnetic spectrometer PRISMA placed at a forward angle, providing good ion identification. The two-neutron transfer channel has been analyzed with a semiclassical model that calculated, in the successive approximation, transitions to $0^{+}$states. It has been shown that the transfer probability for the transition to the excited $0^{+}$state in ${ }^{42} \mathrm{Ca}$, whose wave function 

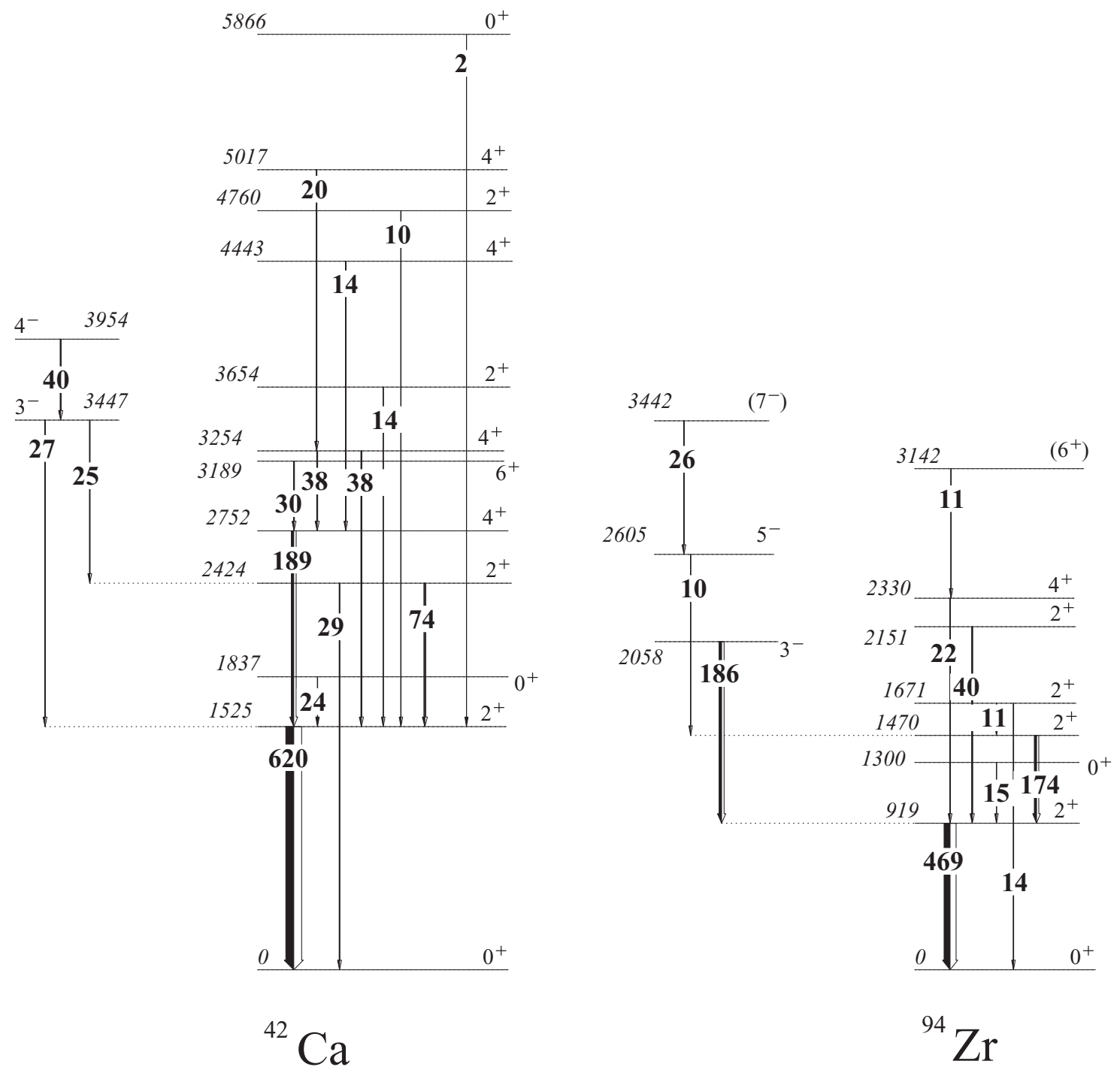

FIG. 8. Level schemes and observed intensities for the ${ }^{42} \mathrm{Ca}$ and ${ }^{94} \mathrm{Zr}$ nuclei reconstructed from the measured $\gamma$ transitions in ${ }^{40} \mathrm{Ca}+{ }^{96} \mathrm{Zr}$ at $E_{\text {lab }}=152 \mathrm{MeV}$ [20]. The experiment has been performed with the PRISMA and CLARA [30] setup. The $\gamma$-ray spectra of binary partners have been obtained after Doppler correction. The correction for the projectilelike ions is based on the knowledge of their velocity vector measured with the spectrometer. The correction of the (undetected) targetlike ions is based on the assumption of binary kinematics and the corresponding calculated velocity. The intensities (reported in the figure as normalized counts, bold numbers) have been obtained taking into account the branching ratios [31] and the efficiency of the CLARA $\gamma$ array.

is dominated by the two neutrons in the $2 p_{3 / 2}$ shell, is much larger than the ground-state one, but by considering only $0^{+}$ transitions the experimental cross section is underpredicted by a factor of $\sim 3$. This is ascribed to transitions to states with large angular momentum and to transitions of non-natural character, indicating that more complex two-particle correlations have to be considered in the transfer process.

It is important to pursue further studies at sub-Coulomb energies in systems with different nuclear structure, in particular for cases where the ground-state $Q$ values match the optimum ones. Of course, one should also try to get information on correlations involving proton transfer channels. These studies will be of particular relevance in the near future due to the advent of radioactive beams where these problematics are of top priority to understand some of the basic properties of very weakly bound nuclei. In this context it is extremely important to extend the formalism in such a way to calculate the transfer of pairs with large angular momentum and where the full recoil is taken into account.

\section{ACKNOWLEDGMENTS}

The authors are grateful to the LNL Tandem-ALPI staff for providing us with the good quality beams and the target laboratory for excellent target preparation. This work was partly supported by the European Community FP6, Structuring the ERA, Integrated Infrastructure Initiative, Contract EURONS No. RII3-CT-2004-506065. This work was also supported in part by the Croatian Ministry of Science, Education and Sports, Grant No. 0098-1191005-2890. 
[1] R. A. Broglia, O. Hansen, and C. Riedel, Advances in Nuclear Physics, edited by M. Baranger and E. Vogt, (Plenum, New York, 1973), Vol. 6, p. 287.

[2] K. D. Dietrich, K. Hara, and F. Weller, Phys. Lett. B 35, 349 (1971).

[3] J. Dobaczewski, I. Hamamoto, W. Nazarewicz, and J. A. Sheikh, Phys. Rev. Lett. 72, 981 (1994).

[4] I. Tanihata et al., Phys. Rev. Lett. 100, 192502 (2008).

[5] G. Potel, F. Barranco, E. Vigezzi, and R. A. Broglia, Phys. Rev. Lett. 105, 172502 (2010).

[6] S. Landowne and H. H. Wolter, Nucl. Phys. A 351, 171 (1981).

[7] R. A. Broglia and A. Winther, Phys. Rep. C 4, 153 (1992).

[8] J. Knoll and R. Schaeffer, Ann. Phys. (NY) 97, 307 (1976).

[9] R. A. Broglia and A. Winther, Heavy Ion Reactions (AddisonWesley Pub. Co., Redwood City, CA, 1991).

[10] B. F. Bayman and J. Chen, Phys. Rev. C 26, 1509 (1982).

[11] E. Maglione, G. Pollarolo, A. Vitturi, R. A. Broglia, and A. Winther, Phys. Lett. B 162, 59 (1985).

[12] J. H. Sørensen and A. Winther, Nucl. Phys. A 550, 306 (1992).

[13] A. H. Wuosmaa, K. E. Rehm, B. G. Glagola, Th. Happ, W. Kutschera, and F. L. H. Wolfs, Phys. Lett. B 255, 316 (1991)

[14] K. E. Rehm, B. G. Glagola, W. Kutschera, F. L. H. Wolfs, and A. H. Wuosmaa, Phys. Rev. C 47, 2731 (1993).

[15] K. E. Rehm, A. M. van den Berg, J. J. Kolata, D. G. Kovar, W. Kutschera, G. Rosner, G. S. F. Stephans, and J. L. Yntema, Phys. Rev. C 37, 2629 (1988).

[16] W. von Oertzen, H. G. Bohlen, B. Gebauer, R. Künkel, F. Pühlhofer, and D. Schüll, Z. Phys. A 326, 463 (1987).
[17] R. Künkel, W. von Oertzen, H. G. Bohlen, B. Gebauer, H. A. Bösser, B. Kohlmeyer, J. Speer, F. Pühlhofer, and D. Schüll, Z. Phys. A 336, 71 (1990).

[18] W. von Oertzen et al., Eur. Phys. J. A 20, 153 (2004).

[19] A. M. Stefanini et al., Nucl. Phys. A 701, 217c (2002).

[20] S. Szilner et al., Phys. Rev. C 76, 024604 (2007).

[21] L. Corradi, G. Pollarolo, and S. Szilner, J. Phys. G: Nucl. Part. Phys. 36, 113101 (2009).

[22] R. R. Betts et al., Phys. Rev. Lett. 59, 978 (1987).

[23] R. B. Roberts, S. B. Gazes, J. E. Mason, M. Satteson, S. G. Teichmann, L. L. Lee Jr., J. F. Liang, J. C. Mahon, and R. J. Vojtech, Phys. Rev. C 47, R1831 (1993).

[24] C. L. Jiang, K. E. Rehm, J. Gehring, B. Glagola, W. Kutschera, M. Rhein, and A. H. Wuosmaa, Phys. Lett. B 337, 59 (1994).

[25] C. L. Jiang, K. E. Rehm, H. Esbensen, D. J. Blumenthal, B. Crowell, J. Gehring, B. Glagola, J. P. Schiffer, and A. H. Wuosmaa, Phys. Rev. C 57, 2393 (1998).

[26] R. A. Broglia, G. Pollarolo, and A. Winther, Nucl. Phys. A 361, 379 (1981).

[27] G. Pollarolo, R. A. Broglia, and A. Winther, Nucl. Phys. A 406, 369 (1983).

[28] R. D. Lawson, Theory of the Nuclear Shell Model (Clarendon Press, Oxford, 1980).

[29] D. C. Williams, J. D. Knight, and W. T. Leland, Phys. Rev. C 164, 1419 (1967).

[30] A. Gadea et al., Eur. Phys. J. A 20, 193 (2004).

[31] B. Singh and J. A. Cameron, Nuclear Data Sheets 92, 1 (2001); D. Abriola and A. A. Sonzogni, ibid. 107, 2423 (2006); National Nuclear Data Center [http://www.nndc.bnl.gov]. 